\title{
Tardy but intact-publication of REVIVE trial results welcomed
}

$\mathrm{R}$ esults from the REVIVE trials, first presented at the 2005 AHA Scientific Sessions, have finally been published. REVIVE I was designed to develop a measure of efficacy for the treatment of patients with acutely decompensated heart failure (ADHF). In REVIVE II, this tool was then used to evaluate the efficacy of levosimendan, a calcium-sensitizing inotropic agent with vasodilatory properties, in patients with ADHF. Although levosimendan provided symptomatic relief for 5 days, the drug was also associated with an increased risk of adverse cardiovascular events within 90 days of treatment.

ADHF symptoms can be difficult to quantify. The trial investigators, therefore, developed an appropriate quantitative tool in REVIVE I, which combined patient global assessments (in which patients characterized their own changes in overall clinical status $6 \mathrm{~h}, 24 \mathrm{~h}$, and 5 days after treatment initialization) with physician assessments to produce the primary end point used in REVIVE II. In their paper, the investigators also highlight that "the clinical status of many patients with ADHF might destabilize in the days and weeks after initial symptom relief," hence they chose to investigate the longer-term impacts of levosimendan.

In REVIVE II, patients admitted to hospital for treatment of ADHF were randomly assigned to receive either levosimendan (intravenous bolus of $12 \mu \mathrm{g} / \mathrm{kg}$ over $10 \mathrm{~min}$ followed by a continuous infusion of $0.1 \mu \mathrm{g} / \mathrm{kg} / \mathrm{min}$ for up to $24 \mathrm{~h}$ ) or placebo. More patients receiving levosimendan had clinical improvement and fewer had clinical worsening than patients receiving placebo $(P=0.015$ for the difference between the groups).

Patients who received levosimendan, however, had a numerically higher rate of death in the 90 days after treatment (HR 1.33, 95\% CI 0.85-2.06, $P=0.21$ ). The increased relative risk of death was higher for patients who were already at increased risk of death before entry into the study. Patients with a pretreatment systolic blood pressure $<100 \mathrm{mmHg}$ had a relative risk of death of 1.9 compared with patients treated with placebo; the relative risk was 1.1 for patients with a systolic blood pressure $\geq 100 \mathrm{mmHg}$.

Five adverse events were more common $(P<0.05$ for each $)$ in patients treated with levosimendan than with placebo: hypotension ( $50 \%$ vs $36 \%$ ), headache (30\% vs $15 \%)$, ventricular tachycardia ( $25 \%$ vs $17 \%)$, atrial fibrillation ( $9 \%$ vs $2 \%$ ) and ventricular extrasystoles ( $8 \%$ vs $2 \%$ ). Skin rashes, however, were more frequent in patients treated with placebo than with levosimendan ( $1 \%$ vs $4 \%$; $P<0.05$ ).

"This publication of the results from REVIVE represents the most definitive dataset about the efficacy and safety of levosimendan in acute heart failure, a drug currently in clinical use in many parts of the world," says Michael Felker from the Duke Heart Center, Durham, NC, USA, who was not involved in the trial. "Although these data have been known to many people working in the field since 2005, they have not been readily accessible in detail until now."

In an editorial published with the trial reports, Robert Califf, also from the Duke Heart Center, welcomed the publication of this trial, which "represents a milestone for the cardiovascular community, who should rejoice in the fact that the REVIVE investigators have finally decided to come out of the data cellar". Califf describes trial investigators and sponsors as having obligations firstly to "the study participants, who signed a consent form waiving some of their freedoms in order to participate in ... a clinical trial whose purpose is explicitly defined by the US Department of Health and Human Services as 'the creation of generalizable knowledge"' Their second obligation, according to Dr Califf, is to "their clinical and scientific colleagues,

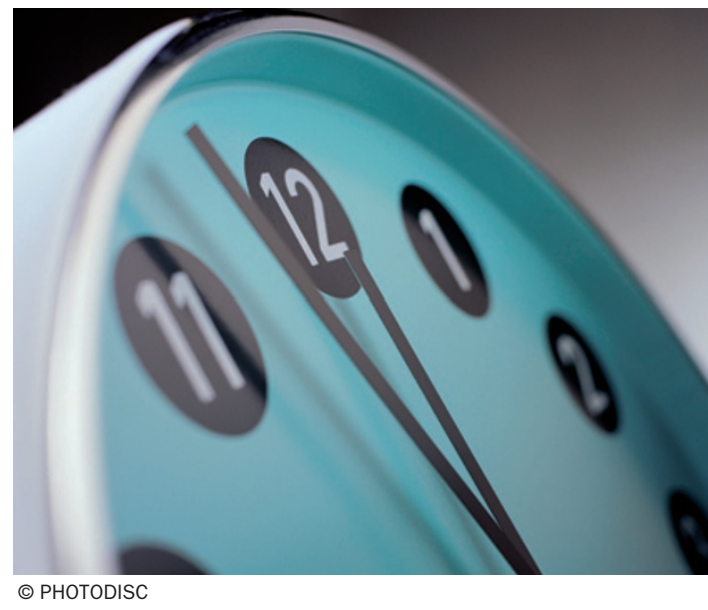

who have been forced to make decisions about drug development and clinical practice in the setting of ADHF in the absence of a complete and accurate peerreviewed accounting of the results derived from these trials".

Christopher O'Connor, editor-in-chief of JACC Heart Failure, where the trial results were published, discussed the complex reasons for "delayed publication of neutral or negative trials" in his Editor's page. He suggests that "if there is no intent to publish 1 year beyond study completion, the Data Safety and Monitoring Board report could form the basis of the published trial results". Biykem Bozkurt, from Baylor College of Medicine, Houston, TX, USA, who was not involved in the study, commends both the editors of JACC Heart Failure and the REVIVE investigators for "embracing the importance of publishing the results of this clinically very relevant and important study, even 8-9 years after [its] completion, and even if the results were not positive or as anticipated. It is known that there usually is a publication bias in clinical trials due to statistical significance or direction of trial results, but it is refreshing to see that this publication breaks that bias."

Megan Cully

Original article Packer, M. et al. Effect of levosimendan on the short-term clinical course of patients with acutely decompensated heart failure. JACC Heart Fail. 1, 103-111 (2013)

Further reading Califf, R. M. Better late than never. JACC Heart Fail. 1, 112-113 (2013) | O'Connor, C. Dead letter office. JACC Heart Fail. 1, 181-182 (2013) 